\title{
The effects of Jiao-Tai-Wan on sleep, inflammation and insulin resistance in obesity-resistant rats with chronic partial sleep deprivation
}

Xin Zou', Wenya Huang ${ }^{1}$, Fuer Lu', Ke Fang ${ }^{1}$, Dingkun Wang ${ }^{1}$, Shuyong Zhao², Jiming Jia², Lijun Xu', Kaifu Wang ${ }^{1}$, Nan Wang ${ }^{3^{*}}$ and Hui Dong ${ }^{1 *}$

\begin{abstract}
Background: Jiao-Tai-Wan (JTW), composed of Rhizome Coptidis and Cortex Cinnamomi, is a classical traditional Chinese prescription for treating insomnia. Several in vivo studies have concluded that JTW could exert its therapeutical effect in insomnia rats. However, the specific mechanism is still unclear. The present study aimed to explore the effect of JTW on sleep in obesity-resistant (OR) rats with chronic partial sleep deprivation (PSD) and to clarify its possible mechanism.
\end{abstract}

Methods: JTW was prepared and the main components contained in the granules were identified by 3D-High Performance Liquid Chromatography (3D-HPLC) assay. The Male Sprague-Dawley (SD) rats underwent $4 \mathrm{~h}$ PSD by environmental noise and the treatment with low and high doses of JTW orally for 4 weeks, respectively. Then sleep structure was analyzed by electroencephalographic (EEG). Inflammation markers including high-sensitivity C reactive protein (hs-CRP), tumor necrosis factor-a (TNF-a) and interleukin-6 (IL-6) levels were examined in the rat plasma. Meanwhile, metabolic parameters as body weight increase rate, fasting plasma glucose (FPG), fasting insulin (FINS) levels and insulin resistance index (HOMA-IR) were measured. The expressions of clock gene cryptochromes (Cry1 and Cry2) and inflammation gene nuclear factor-KB (NF-KB) in peripheral blood monocyte cells (PBMC) were also determined.

Results: The result showed that the administration of JTW significantly increased total sleep time and total slow wave sleep (SWS) time in OR rats with PSD. Furthermore, the treatment with JTW reversed the increase in the markers of systemic inflammation and insulin resistance caused by sleep loss. These changes were also associated with the up-regulation of Cry 1 mRNA and Cry 2 mRNA and the down-regulation of NF-kB mRNA expression in PBMC.

Conclusions: This study suggests that JTW has the beneficial effects of improving sleep, inflammation and insulin sensitivity. The mechanism appears to be related to the modulation of circadian clock and inflammation genes expressions in PBMC.

Keywords: Jiao-Tai-Wan, Sleep deprivation, Inflammation, Insulin resistance, Circadian clock

\footnotetext{
* Correspondence: southernwang@sina.com; tjhdonghui@163.com

${ }^{3}$ Department of Radiology, Tongji Hospital, Tongji Medical College, Huazhong University of Science and Technology, Wuhan 430030, People's Republic of China

'Institute of Integrated Traditional Chinese and Western Medicine, Tongii Hospital, Tongji Medical College, Huazhong University of Science and Technology, Wuhan 430030, People's Republic of China

Full list of author information is available at the end of the article
} 


\section{Background}

Sleep loss has become a global problem due to socialeconomic factors in the modern society. Since sleep is important to recover our energy, inadequate sleep is always thought to be a reason for weight loss [1]. However, growing evidence suggests that sleep curtailment is a new risk factor for the development of obesity and type 2 diabetes [2, 3]. For instance, in the United States, self-reported sleep duration has decreased by $1.5 \sim 2 \mathrm{~h}$ in the last 50 years coinciding with an increase in obesity and diabetes [4]. In China, nearly one-third of the population reported poor sleep quality or shortened sleep duration $(\leq 6 \mathrm{~h})$. Compared to subjects with good quality sleep who slept for 6-8 h per night, those who have poor sleep quality and short sleep duration were more likely to have diabetes [5].

With regard to the pathophysiology of sleep lossassociated obesity and diabetes, present literature highlights the role of inflammation and insulin resistance (IR) after sleep curtailment [6]. Clinical studies have found that plasma inflammatory cytokines increase significantly with the activation of cellular inflammation [7]. These alterations could be observed even after one night of sleep deprivation [8]. The existence of chronic low-grade inflammation may be associated with IR and precedes the onset of type 2 diabetes in adults [9]. Thus getting enough good sleep plays an important role of maintaining metabolic health. In addition, insomnic patients often take sedative-hypnotics, antipsychotics, antidepressants and anti-anxiety medications. Abnormal long sleep duration and increased appetite associated with these drugs may also cause insulin resistance and high risk of weight-gain. Therefore, the therapy with a dual-effect of sleep and IR improvement is promising [10].

Jiao-Tai-Wan (JTW), composed of Rhizome Coptidis (Coptis chinensis Franch, Ranunculaceae) and Cortex Cinnamomi (Cinnamomum cassiaPresl, Lauraceae), is a classical traditional Chinese prescription for treating insomnia [11]. The application of JTW to treat insomnia can be traced back to Han Shi Yi Tong in Ming Dynasty. The function of Rhizome Coptidis (Huanglian in Chinese) is to clear away heart fire and the function of Cortex Cinnamomi (Rougui in Chinese) is to warm kidney water. According to a survey, Rhizome Coptidis was one of the top 10 individual Chinese herbs prescribed for insomnia in Taiwan during 2002 [12]. In a meta-analysis within 217 reviewed studies, JTW was one of the 10 most frequently examined standardized Chinese herbal formulas for insomnia [13]. In in vivo studies, JTW has been proven to play its sedative and hypnotic role on insomnia rats $[14,15]$. Previous researches have demonstrated that JTW also exhibits hypoglycemic effect and insulin sensitizing activity in diabetic rodents and diabetic patients $[16,17]$. The possible mechanisms may be related to suppressing gluconeogenesis by activating adenosine $5^{\prime}$-monophosphate-activated protein kinase in the liver and enhancing insulin signaling through phosphatidylinositol 3-kinase pathway in the skeletal muscle $[18,19]$. Given that sleep loss induces inflammation and IR, we hypothesize that JTW may have the potential to alleviate inflammation and improve insulin sensitivity after sleep curtailment. Also, one study suggested that multiple-dose oral administration of JTW may improve its absorption in insomnic rats compared with normal rats, which will increase the bioavailability, and give full play of its therapeutical effect [20]. Therefore, we established a rat model of chronic partial sleep deprivation (PSD) and investigated the effects and mechanisms of JTW on sleep structure, inflammation and IR.

\section{Methods}

\section{Animals}

Male Sprague-Dawley (SD) rats $(n=250,10$ weeks, 200 \pm 20 g) were supplied by Hubei Province Center for Disease Control and Prevention (Wuhan, China). The rats were maintained on $12 \mathrm{~h}$ light-12 h dark cycle in a humidity- and temperature-controlled environment. All procedures were approved by Huazhong University of Science and Technology Ethics Committee for the use of experimental animals (IRB ID: TJ-A20131218). After fed with food and water ad libitum for 1 week, all rats were switched to a high-fat, high-energy diet (8\% corn oil, 44\% sweetened condensed milk, 48\% standard rat chow) for 2 weeks. Then 100 rats with the lowest weight gain (about 40\%) were classified as obesity-resistant (OR) rats [21]. As has been previously proved, better sleep quality may confer obesity protection in OR rats [22]. However, chronic partial sleep deprivation increases body weight even in OR rats which is mostly consistent with human conditions [23]. Accordingly, OR rat is an excellent animal model to study sleep loss-associated obesity and IR [24].

\section{Preparation of JTW}

JTW is composed of Rhizome Coptidis and Cortex Cinnamomi. The ratio of these two herbs is 10:1 (w/w). In the present study, we use Rhizome Coptidis and Cortex Cinnamomi concentrated granules (purchased from China Resources Sanjiu Medical and Pharmaceutical Co., Ltd) for rat administration. Rhizome Coptidis and Cortex Cinnamomi were all purchased from Rui Sheng Yuan Biotechnology Co., Ltd. (Chengdu, China) and identified by Department of Chinese Medicine Authentication of Jin Chan Pharmaceutical Co., Ltd. (Hefei, China). All the samples are deposited in the herbarium of the same department with voucher number No. 472 for Rhizoma Coptidis, No. 353 for Cortex 
Cinnamomi. The herbs were extracted by using a method of simulated family decoction with boiling water. Then the extracts were concentrated, isolated, dried to form granules. This process of production was performed according to Good Manufacturing Practice (GMP) for Drugs (Chinese FDA, 2010 Version) to ensure the quality control. And the obtained products were re-identified by Guangdong Institute for Food and Drug Control (Guangzhou, China). As a result, $0.5 \mathrm{~g}$ of Rhizome Coptidis granule efficacy is equivalent to $3 \mathrm{~g}$ decoction pieces and $1 \mathrm{~g}$ of Cortex Cinnamomi granule efficacy is equivalent to $3 \mathrm{~g}$ decoction pieces. We mixed the granules together by distilled warm water and administered to the rat at the decoction (not granule) pieces dosage of $1.1 \mathrm{~g} / \mathrm{kg}$ and $2.2 \mathrm{~g} / \mathrm{kg}$ body weight/day, respectively. The calculation method was as follows: for a person with body weight of $60 \mathrm{~kg}$, the high dose of daily JTW intake is $22 \mathrm{~g}$ (20 g of Rhizoma Coptidis and $2 \mathrm{~g}$ of Cortex Cinnamomi). According to the conversion of human doses (Chinese Pharmacoepia, 2010) to rat equivalent doses based on body surface areas, the daily dose of JTW intake in rats is 6 times higher than human. Therefore, we chose $2.2 \mathrm{~g} / \mathrm{kg}$ body weight as the daily dose by gavage to the rat.

\section{Determination of the main chemical constituents in JTW by 3D-HPLC analysis}

The main chemical constituents in JTW were identified by 3D-High Performance Liquid Chromatography (3DHPLC) method (Fig. 1a). The reference standards of cinnamaldehyde, cinnamon acid, palmatine chloride and berberine hydrochloride were purchased from National Institutes for Food and Drug Control (Beijing, China). The reference standards of coptisine hydrochloride and jatrorrhizine hydrochloride were purchased from Tauto Biotechnology Ltd. (Shanghai, China) (Fig. 1b). For the analysis, the drying powder Rhizome Coptidis (0.25 g) and Cortex Cinnamomi (0.05 g) were accurately weighed, mixed and extracted with $100 \mathrm{ml}$ of methanol in an ultrasonic bath for $60 \mathrm{~min}$. Additional methanol was added to compensate for any lost volume. Then the resulting solution was filtered through a $0.45 \mu \mathrm{m}$ membrane filter prior to HPLC injection. HPLC was performed on a Waters 2695-2996HPLC system (Waters Corporation, Milford, MA, USA) equipped with an Agilent TC-C18 column $(4.6 \mathrm{~mm} \times 250 \mathrm{~mm}$, 5um). The mobile phase included acetonitrile (A) and $0.05 \mathrm{~mol} / \mathrm{l}$ potassium dihydrogen phosphate (B) at a flow speed of $1.0 \mathrm{ml} / \mathrm{min}$ in the condition of column temperature $30{ }^{\circ} \mathrm{C}$. The detection wavelength was set at $270 \mathrm{~nm}$ and the sample injection volume was $10 \mu \mathrm{l}$. The gradient elution was as follows: $0 \sim 5 \mathrm{~min}, 85 \% \sim 85 \% \mathrm{~B}, 5 \sim 40 \mathrm{~min}, 85 \% \sim 57 \%$ B. Chromatographic data was collected and analyzed by using Waters Empower 3 software. Moreover, ten batches of JTW which were supplied by China Resources Sanjiu Medical and Pharmaceutical Co., Ltd. were used to detect the similarity (Fig. 1c). The similarity of fingerprints was analyzed by professional software named Similarity Evaluation System for Chromatogram Fingerprint of Traditional Chinese Medicine (version of 2004A).

\section{Experimental design}

Selected OR rats were switched to standard laboratory chow and randomly allocated to four groups, control group, model group, low dose JTW treated group (JTW1) and high dose JTW treated group (JTW2) (25 rats in each group). Rats in model group and JTW treated group were subjected to PSD during the light phase ( 4 h/day from 10:00 to 14:00) for 4 weeks. Sleep deprivation was achieved by exposing the animals to environmental noise, as previously described [23]. Meanwhile, rats in JTW1 and JTW2 treated group were respectively given low and high doses of JTW granules by intragastric administration once a day. Five rats in each group were sacrificed to collect blood samples at the 1st, 2nd, 3rd and 4th week. Blood was collected from aorta abdominalis for biochemical analysis and Peripheral Blood Mononuclear Cells (PBMC) isolation.
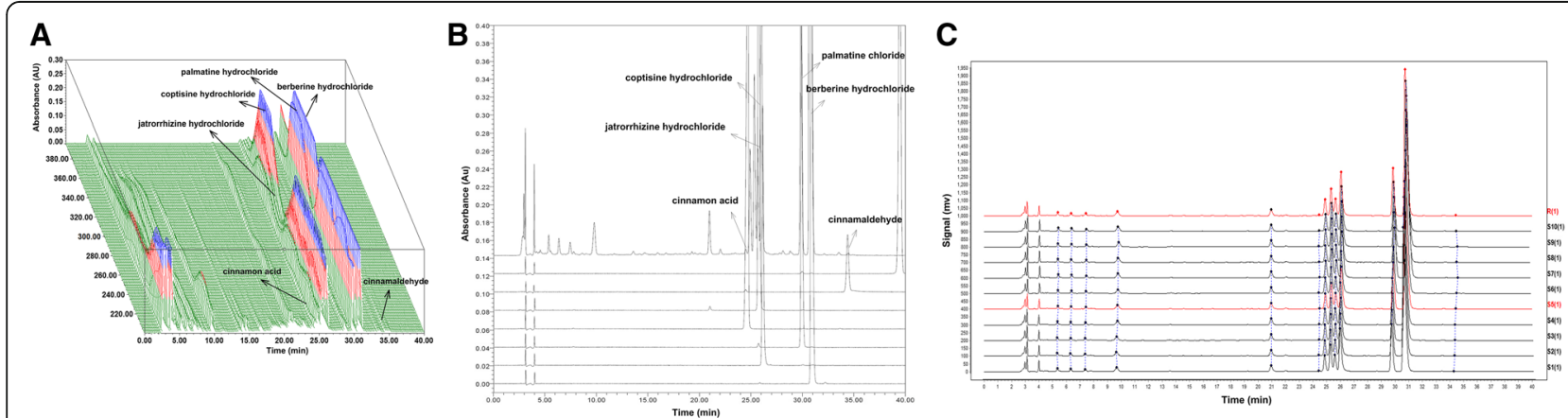

Fig. 1 The 3D-HPLC chromatogram of JTW. a 3D-HPLC fingerprint of JTW; b HPLC chromatograms of six standard chemicals; $\mathbf{c}$ HPLC chromatogram from ten different batches of JTW to detect the similarity. Standardization of JTW was done using HPLC fingerprinting with chemical standards including cinnamon acid, jatrorrhizine hydrochloride, coptisine hydrochloride, palmatine hydrochloride, berberine hydrochloride, cinnamaldehyde 


\section{Surgery, sleep recordings and analysis}

Another five rats in each group underwent surgery 1 week prior to any PSD subjection or JTW intake, using standard procedures as bellow [25]. Under pentobarbital (50 $\mathrm{mg} / \mathrm{kg}$, intraperitoneal injection)-induced anesthesia, two stainless steel screws (2 mm long, $1.2 \mathrm{~mm}$ of diameter) attached to insulated wire were implanted in the skull over the frontal-parietal cortex for electroencephalographic (EEG) recordings. One of the electrodes was placed approximately $2 \mathrm{~mm}$ anterior and $5 \mathrm{~mm}$ to the right of bregma; another was placed approximately $2 \mathrm{~mm}$ anterior and $5 \mathrm{~mm}$ to the left of bregma. Both of the electrodes were attached to a miniature connector. All connectors were cemented in place with dental acrylic. Right after the surgical implantation, the rats were injected with Penicillin sodium (80,000 unit dose for each rat) for 2 days and allowed to recover for 7 days prior to the initiation of the experiments. At the end of the 4th week, EEG recordings were collected. For the purpose of habituation, the animals were connected to the recording apparatus at least 1 day before the experiments for EEG recordings. The miniature connectors were connected to a RM-6280C 8-channel physiological signal recorder (Chengdu Instrument Factory, Chengdu, China) via the signal input lines. Make sure the animal can move freely within a wide range. The signals were amplified and filtered $(1 \sim 30 \mathrm{~Hz})$ digitized at a speed of $200 \mathrm{~ms} / \mathrm{disc}$, and recorded using Model RM6280C physiological signal acquisition and processing system. The system was run for EEG recording continuously for $4 \mathrm{~h}$ (10:00-14:00). The EEG waveform files were saved and the waves ratio tables were exported for further analysis. The power spectrum densities, integrated and averaged, could be divided into four frequency areas: $\delta$ wave $(0.5 \sim 4 \mathrm{~Hz}), \theta$ wave $(4 \sim 8 \mathrm{~Hz})$, $\alpha$ wave $(8 \sim 14 \mathrm{~Hz})$, and $\beta$ wave $(14 \sim 30 \mathrm{~Hz})$. According to rat EEG, each epoch was assigned to one of the following categories [26]: wakefulness (can be divided into two types of EEG: $\theta$ wave $(4 \sim 8 \mathrm{~Hz})$ when the rats in walking, climbing, exploring and focus behavior; irregular high-frequency and low-amplitude EEG activity when the rats licking the body and standing still), slow-wave sleep stage I ( $\mathrm{SWS}_{1}$, high-amplitude, low-frequency $(1 \sim 5 \mathrm{~Hz})$ and spindle waves $(8 \sim 14 \mathrm{~Hz})$ synchronous pattern activity, and high-amplitude and low-frequency wave ratio less than $50 \%$ share in each of the statistical unit (30 s), eyes closed, usually lying on the animal's side or curled up with head down), slow-wave sleep stage II $\left(\mathrm{SWS}_{2}\right.$, same as $\mathrm{SWS}_{1}$, but high-amplitude and low-frequency wave ratio more than $50 \%$ share in each of the statistical unit), REM sleep (low-frequency $\theta$ wave, with no significant difference between the waking state, occasional body twitches while maintaining a recumbent sleep posture. Since wakefulness could not directly turn to the REM sleep state, there was always the presence of SWS sleep before the REM sleep, and REM sleep could be directly convert into wakefulness or SWS sleep. REM sleeping time generally sustained not more than $3 \mathrm{~min}$ ). Each individual sleeping phase lasted at least $20 \mathrm{~s}$, and each analysis unit was $30 \mathrm{~s}$. The total sleep time, total time of slow wave sleep $\left(\mathrm{SWS}_{\text {total }}\right), \mathrm{SWS}_{1}$ and $\mathrm{SWS}_{2}$, and total time of REM sleep were calculated to analyze the differences among the four groups.

\section{Determination of plasma hs-CRP, TNF- $\alpha$ and IL- 6 levels}

Plasma high-sensitivity $C$ reactive protein (hs-CRP), tumor necrosis factor- $\alpha$ (TNF- $\alpha$ ) and interleukin-6 (IL6) levels were determined using the ELISA kits (Boster Bio-engineering Co., Ltd., Wuhan, China), according to the manufacturer's protocol.

\section{Measurement of body weight increase rate, FPG, FINS levels and HOMA-IR}

The body weight increase rate (\%) was calculated by the net weight increase ( $\mathrm{g}$ ) each week divided by the previous weight (g). Fasting plasma glucose (FPG) was measured by the glucose oxidase method using a commercially available kit (Mingdian Bio-engineering Co., Ltd., Shanghai, China) and fasting insulin (FINS) level was determined by ELISA method (Boatman Biotech Co., Ltd., Shanghai, China). The homeostasis model assessment index of IR (HOMA-IR) was calculated using the formula of FPG $(\mathrm{mmol} / \mathrm{L}) \times$ FINS $(\mu \mathrm{IU} / \mathrm{mL}) / 22.5$.

\section{Assessment of clock gene Cry and inflammation gene NF-кB expression in PBMC}

The PBMC in different groups was isolated by using a Ficoll density gradient separation kit (Haoyang Biological Manufacture Co., Ltd., Tianjin, China) as previously described [27]. The resulting cells were re-suspended in $1 \mathrm{ml}$ Trizol (Ambion Inc., Austin, TX, USA) and stored at $-80{ }^{\circ} \mathrm{C}$. Total RNA was extracted according to the manufacturer's instructions. The purity and concentration of the extracted RNA were measured by a Nucleic Acid/Protein Analyzer (Thermo, Rockford, USA). Then $1 \mu \mathrm{g}$ of total RNA was reverse-transcribed using a Prime Script RT reagent Kit in a total reaction volume of $20 \mu \mathrm{l}$. cDNA was synthesized in a Mastercycler gradient PCR apparatus (Eppendorf Company, Hamburg, Germany). Then $2.0 \mu \mathrm{l}$ of this cDNA was amplified in a $20 \mu \mathrm{l}$ PCR reaction mixture containing $6.8 \mu \mathrm{lddH} 2 \mathrm{O}, 0.4 \mu \mathrm{l}$ forward primer, $0.4 \mu \mathrm{l}$ reverse primer, $0.4 \mu \mathrm{l}$ ROX Reference Dye $(50 \times)$ and $10.0 \mu \mathrm{l} \mathrm{SYBR}$ premix EX Taq ${ }^{\mathrm{Ta}}(\mathrm{TaKaRa}$ Company, Dalian, China) with an Applied Biosystems StepOne Real-Time PCR System (StepOne, Foster City, USA). The reaction included 40 cycles of the following 3 stages: stage $1,95^{\circ} \mathrm{C}$ for $30 \mathrm{~s}$; stage $2,95^{\circ} \mathrm{C}$ for $5 \mathrm{~s}$,; stage $3,60{ }^{\circ} \mathrm{C}$ for $30 \mathrm{~s}$. The primer sequences were shown in 
Table 1. The method of $2^{-\Delta \Delta C T}$ was used to calculate the relative expression level of each group.

\section{Statistical analysis}

Data were presented as mean \pm standard deviation (SD) and analyzed using SPSS 19.0 software. One-way analysis of variance (ANOVA) was used to determine the statistical significance, followed by a LSD post hoc test (for equal variances) or a Dunnett's T3 post hoc test (for not assumed equal variances). $p<0.05$ was considered statistically significant.

\section{Results}

\section{D-HPLC profile of JTW}

3D-HPLC chromatogram of JTW was shown in Fig. 1a. Six of the constituents in JTW were identified by comparing the retention time and peak height with reference standard (Fig.1b). The main constituents of JTW were as follows: cinnamon acid, jatrorrhizine hydrochloride, coptisine hydrochloride, palmatine chloride, berberine hydrochloride and cinnamaldehyde. HPLC chromatogram of JTW from ten batches was shown in Fig. 1c and the similarity coefficients were no less than 0.99

\section{The effect of JTW on sleep parameters in sleep- deprivated OR rats}

As shown in Table 2, during the $4 \mathrm{~h}$ EEG recording condition, total sleep time and total SWS time were significantly reduced in OR rats with PSD $(p<0.01)$. Time spent in $\mathrm{SWS}_{1}$ and $\mathrm{SWS}_{2}$ were also decreased $(p<0.01)$. However, JTW1 and JTW2 treatment increased total sleep time, $\mathrm{SWS}_{\text {total }}$ time, especially $\mathrm{SWS}_{1}$ time in OR rats with PSD $(p<0.01)$. And JTW1 and JTW2 slightly increased the $\mathrm{SWS}_{2}$ time compared with the model group. As for REM time, there was no significant difference among these four groups (data not shown). The result suggests that JTW has a beneficial effect on extending the total sleeping time, especially $\mathrm{SWS}_{1}$ time in OR rats with PSD, changing the structure to a better state more suitable for sleeping.

Table 1 Real-time PCR primer sequences for Cry1, Cry2 and $\mathrm{NF}-\mathrm{KB}$

\begin{tabular}{|c|c|c|}
\hline Gene & Forward $\left(5^{\prime} \rightarrow 3^{\prime}\right)$ & Reverse $\left(5^{\prime} \rightarrow 3^{\prime}\right)$ \\
\hline Cry1 & $\begin{array}{l}\text { 5'-CAGCCAGCTGACGTG } \\
\text { TTTCC-3' }\end{array}$ & $\begin{array}{l}\text { 5'-AATGCGCACGATGACT } \\
\text { TCCA-3' }\end{array}$ \\
\hline Cry2 & $\begin{array}{l}\text { 5'-ACCGCCTGTGGGACT } \\
\text { TGTA-3' }\end{array}$ & $\begin{array}{l}\text { 5'-TCGCCATAGGAGTTGT } \\
\text { CCAAATA-3' }\end{array}$ \\
\hline$N F-k B$ & $\begin{array}{l}\text { 5'-GAGGGACGACACCTC } \\
\text { TACACATA-3' }\end{array}$ & $\begin{array}{l}\text { 5'-CCCAAGAGTCGTCCA } \\
\text { GGTCA-3' }\end{array}$ \\
\hline$\beta$-actin & $\begin{array}{l}5^{\prime} \text {-AGCCATGTACGTAGC } \\
\text { CATCC-3' }\end{array}$ & $\begin{array}{l}\text { 5'- CTCTCAGCTGTGGTG } \\
\text { GTGAA-3' }\end{array}$ \\
\hline
\end{tabular}

Table 2 The effects of JTW on sleep structure in sleepdeprivated $O R$ rats

\begin{tabular}{lllll}
\hline Groups & $\begin{array}{l}\text { Total sleep } \\
\text { time }(\mathrm{s})\end{array}$ & \multicolumn{3}{l}{ Slow wave sleep $(\mathrm{s})$} \\
\cline { 3 - 5 } & $\mathrm{SWS}_{\text {total }}$ & $\mathrm{SWS}_{1}$ & $\mathrm{SWS}_{2}$ \\
\hline Control & $14,100 \pm 198$ & $14,076 \pm 231$ & $7650 \pm 1672$ & $6426 \pm 1640$ \\
Model & $6492 \pm 486^{\# \#}$ & $6471 \pm 485^{\# \#}$ & $4467 \pm 387^{\# \#}$ & $2004 \pm 730^{\# \#}$ \\
JTW1 & $10,450 \pm 308^{* *}$ & $10,380 \pm 314^{* *}$ & $7600 \pm 273^{* *}$ & $2780 \pm 152$ \\
JTW2 & $12,513 \pm 385^{* *}$ & $12,486 \pm 434^{* *}$ & $9534 \pm 402^{* *}$ & $2952 \pm 276$ \\
\hline
\end{tabular}

Data are expressed as mean \pm SD $(s, n=5)$. SWS total: total slow wave sleep time; $\mathrm{SWS}_{1}$ : slow-wave sleep stage I time; $\mathrm{SWS}_{2}$ : slow-wave sleep stage II time. ${ }^{\# \#} p<0.01$ vs. control group, ${ }^{* *} p<0.01$ vs. model group

\section{sleep-deprivated OR rats}

As shown in Fig. 2a-c, plasma hs-CRP, TNF- $\alpha$ and IL-6 levels were significantly elevated $(p<0.05, p<0.01)$ each week in OR rats with PSD, indicating systemic inflammation after sleep curtailment. Treatment with JTW1 and JTW2 exhibited a decrease in the level of plasma inflammatory biomarkers compared with the model group. This reduction trend was observed throughout the deprivation period and reached statistical significance at the end of the fourth week $(p<0.05, p<0.01)$. In addition, plasma TNF- $\alpha$ level was markedly lower $(p<$ $0.05)$ in JTW2-treated rats at the end of the third week compared to that in model rats (Fig. 2b). IL-6 level was also lower at the end of the second week $(p<0.01)$ in JTW2-treated rats (Fig. 2c). The result suggests that JTW has a beneficial effect on alleviating sleep-loss related inflammation in OR rats.

\section{The effect of JTW on metabolic parameters in sleep- deprivated OR rats}

The body weight increase rate each week was markedly higher $(p<0.01)$ in model rats than that in control rats (Fig. 3a). It indicates that partially sleep-deprivated OR rats gained more weight during the whole sleep deprivation period. Similar changes were also found in the levels of FINS and HOMA-IR (Fig. 3c-d). However, partial sleep deprivation caused a slight elevation in FPG in model rats and FPG level was significantly higher $(p<$ 0.05) than that in control rats at the end of the fourth week (Fig. 3b). Compared with model rats, JTW1 and JTW2-treated rats showed the decreases in body weight increase rate $(p<0.05)$, and JTW2 administration significantly decreased FPG, FINS and HOMA-IR $(p<0.05)$. The differences reached statistical significance at the end of the third or the fourth week $(p<0.05)$ (Fig. 3a-d). The result suggests that chronic sleep deprivation is often associated with insulin resistance which can be attenuated after the intervention of JTW2. 

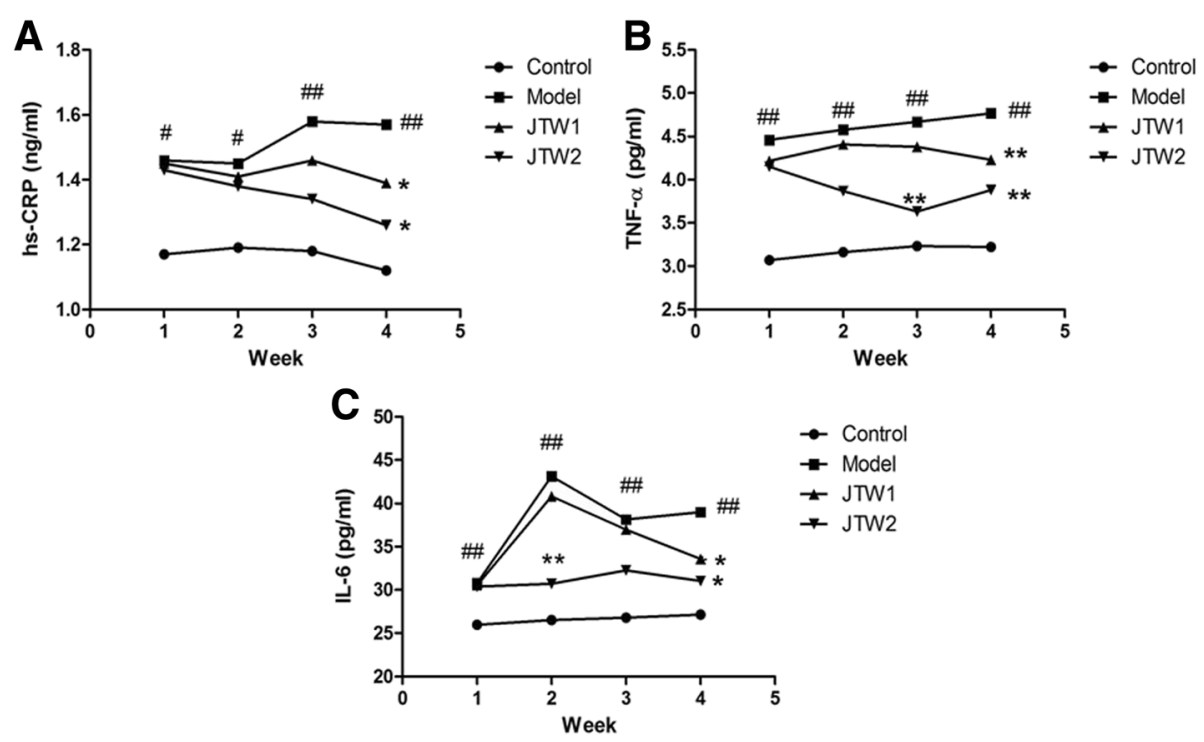

Fig. 2 The effect of JTW on plasma inflammatory biomarkers in sleep-deprivated OR rats. JTW supplementation decreased the level of plasma inflammatory biomarkers in OR rats with PSD (a hs-CRP; b TNF-a; $\mathbf{c} I L-6) .{ }^{\#} p<0.05,{ }^{\# \#} p<0.01$, significantly different from control. ${ }^{*} p<0.05$, ${ }^{* *} p<0.01$, significantly different from model group. Each point represents the mean of 5 rats

The effect of JTW on Cry1mRNA,Cry2mRNA and NFKBmRNA expressions in PBMC of sleep-deprivated OR rats As previously reported, clock protein Cry and inflammation mediator NF- $\mathrm{KB}$ both regulate the expression of proinflammatory cytokines $[28,29]$. The activation of NF- $\mathrm{kB}$ signaling pathway is also involved in the development of insulin resistance [30]. Thus we measured Cry1mRNA, Cry2mRNA and NF- $k$ BmRNA expressions in PBMC of sleep-deprivated OR rats. At the end of the fourth week, the CrylmRNA and Cry2mRNA expressions were markedly decreased while the NF-кBmRNA expression was increased in PBMC of model rats compared with those in control rats $(p<0.01)$ (Fig. $4 \mathrm{a}-\mathrm{c})$. However, the administration of JTW1 and JTW2 reversed the above changes followed by PSD $(p<0.01) \quad$ (Fig. 4a-c). The results suggest that the beneficial effects of JTW on inflammation and insulin resistance may be associated with
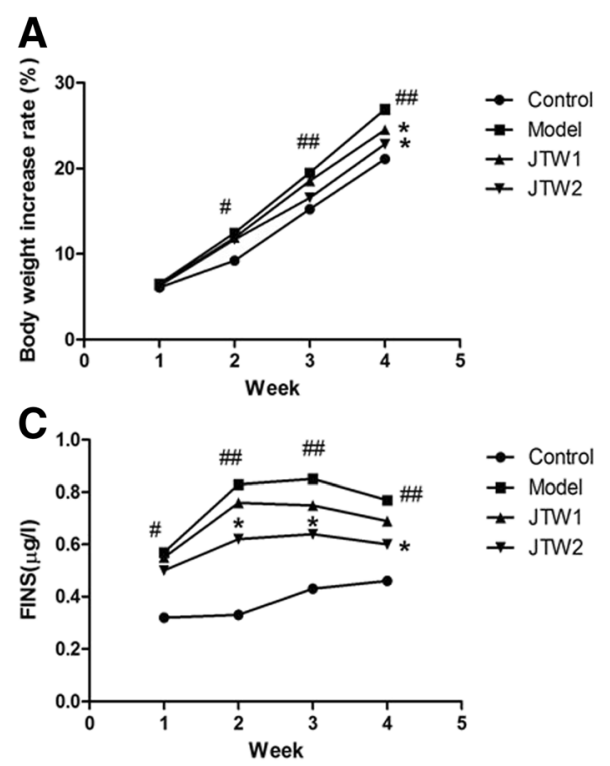

B
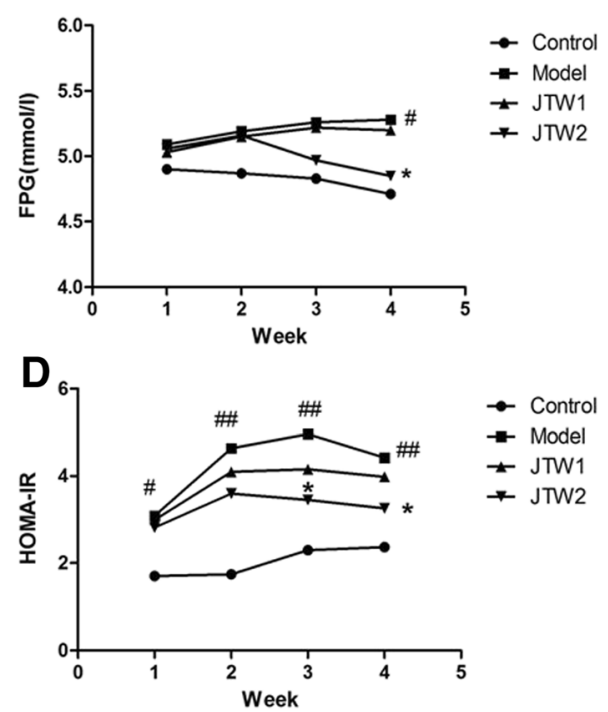

Fig. 3 The effect of JTW on metabolic parameters in sleep-deprivated OR rats. JTW treatment decreased the level of metabolic parameters in OR rats with PSD (a Body weight increase rate; b FPG; c FINS; d HOMA-IR). ${ }^{\#} p<0.05,{ }^{\# \#} p<0.01$, significantly different from control. ${ }^{*} p<0.05$, significantly different from model group. Each point represents the mean of 5 rats 


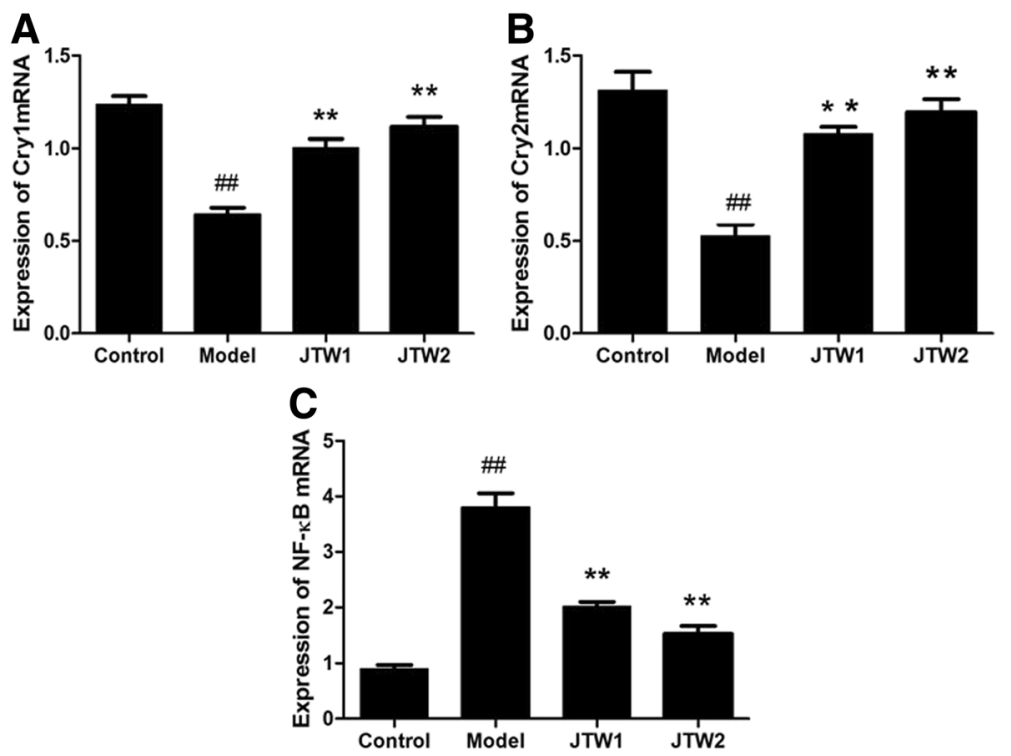

Fig. 4 Effects of JTW on Cry1, Cry2, NF-KB mRNA expressions in PBMC of sleep-deprivated OR rats. JTW treatment increased the level of Cry1mRNA, Cry2mRNA expressions and decreased the level of NF-KBmRNA expression in PBMC of sleep-deprivated OR rats (a Cry1mRNA; b Cry2mRNA; c NF-KBmRNA). ${ }^{\# \#} p<0.01$, significantly different from control. ${ }^{* *} p<0.01$, significantly different from model group. Each point represents the mean of 4 rats

the modulation of circadian clock and inflammation genes expressions in PBMC.

\section{Discussion}

Traditional Chinese medicine has been used to treat insomnia for a long time during Chinese history. However, the underlying mechanism remains unclear. In the present study, we explored the effects and mechanism of JTW on sleep in OR rats with chronic PSD. The result showed that JTW could significantly improve sleep quality in OR rats after sleep curtailment. JTW could also attenuate sleep loss-related inflammation and metabolic disorders. These effects may be associated with the modulation of circadian clock and inflammation genes expressions. All the experimental data indicate that JTW has an obvious hypnotic effect and possesses antiinflammation effect and insulin sensitizing activity.

Human beings keep wakefulness during the day and sleep at night. This sleep/wake cycle is under the control of circadian clock. Circadian clock is the body's biological rhythm in keeping with the 24-h day-night cycle or light-dark transitions. In mammals, the circadian timekeeping system is composed of a central clock in the brain's suprachiasmatic nuclei $(\mathrm{SCN})$ and peripheral clocks in nearly every body cell [31]. At the core of this clock network is a set of interacting transcriptional activators (CLOCK and BMAL1) and repressors (Period and Cryptochrome) [32]. Recent study has found that clock protein Cryptochrome (Cry1 and Cry2) regulates the expression of proinflammatory cytokines. The absence of
Cry1 and Cry2 might elevate intracellular cAMP level and activate protein kinase A (PKA), leading to NF- $\mathrm{kB}$ activation through phosphorylation of p65 at S276 [28]. $\mathrm{NF}-\mathrm{KB}$ is one of the most important regulators of various proinflammatory genes expression. Synthesis of inflammatory cytokines, such as TNF- $\alpha$, IL- $1 \beta$ and IL- 6 , is all mediated by NF- $\mathrm{kB}$. The activated NF- $\mathrm{kB}$ enters the nucleus where it binds to target DNA elements and enhances the transcription of inflammatory genes [29]. This may partially explain the link between circadian rhythm disruption and increased susceptibility to chronic inflammatory diseases.

In the present study, environmental noise seriously disturbed the sleep architecture in OR rat, manifesting as the reduction of total sleep time and total SWS time. SWS plays an important role in glucose regulation and the suppression of SWS may result in decreased insulin sensitivity, leading to impaired glucose tolerance and increased diabetes risk [33]. In addition, environmental noise caused a marked decrease in the expressions of clock gene Cry1mRNA and Cry2mRNA and the increase in the expression of NF- $\mathrm{kB}$ mRNA in PBMC, indicating the disruption of the circadian rhythm and the activation of inflammation. As a result, plasma TNF- $\alpha, \mathrm{IL}-1 \beta$ and IL-6 levels were increased since these inflammatory cytokines are all secreted from PBMC [34]. However, JTW treatment increased total sleep time and total SWS time in OR rats with PSD while decreased plasma inflammatory cytokines levels. It suggested that JTW has the beneficial effects on sleep loss and the sleep-loss 
related inflammation. These effects may be associated with the regulation of the expression of CrylmRNA, Cry2mRNA and NF- $\mathrm{kB}$ mRNA.

In addition, chronic PSD by environmental noise led to a significant weight gain and insulin resistance in OR rats, which is consistent with previous report [23]. In Mavanji's study, the model rats gained more weight than non-deprivated controls after $8 \mathrm{~h}$ PSD daily for 9 days. They also found that poor-quality sleep during rest period leads to enhanced food intake and this may contribute to the weight gain in OR rats. Since we did not record the total and daily food intake in the rats, it is lack of evidence to support this idea. This is also one limitation of our study. However, compared with Mavanji's method, we administered a milder sleep deprivation using a less stressful method (4 h PSD daily for 28 days) to the rats. And we measured the changes of systemic inflammatory condition and metabolic markers which were absent in Mavanji's study. The result showed that sleep curtailment led to a remarkable increase in body weight, FPG, FINS and HOMA-IR. It may attribute to sleep loss-associated inflammation since there is evidence to suggest that chronic low-grade inflammation is a link between obesity and metabolic syndrome. Many studies have proved that inflammatory cytokines such as TNF- $\alpha$ and IL- 6 could decrease insulin sensitivity by negatively affecting the insulin signaling pathway $[35,36]$. However, JTW treatment reversed weight gain and improved insulin sensitivity in OR rats with PSD which may be related to its anti-inflammatory effect.

\section{Conclusions}

In conclusion, our study successfully established a rat model of sleep loss, inflammation and insulin resistance. We also demonstrated that JTW improves sleep, inflammation and insulin sensitivity. The therapeutic effect of JTW may mainly be derived from its modulation of circadian clock and inflammation genes expressions in PBMC.

\footnotetext{
Abbreviations

Cry: Cryptochrome; EEG: Electroencephalographic; FINS: Fasting insulin; FPG: Fasting plasma glucose; HOMA-IR: HOMA insulin resistance index; HPLC: High performance liquid chromatography; hs-CRP: High-sensitivity C reactive protein; IL-6: Interleukin-6; IR: Insulin resistance; JTW: Jiao-Tai-Wan; NF-kB: Nuclear factor-kB; OR: Obesity-resistant; PBMC: Peripheral blood monocyte cells; PSD: Partial sleep deprivation; SWS: Slow wave sleep; TNF-a: Tumor necrosis factor-a
}

\section{Acknowledgments}

Not applicable.

\section{Funding}

This work was supported by grants from National Natural Science Foundation of China (No. 81373871, 81473637).

\section{Availability of data and materials}

All the data is contained in the manuscript.

\section{Authors' contributions}

$H D$ and $X Z$ wrote the study protocol and drafted this manuscript. HD and NW participated in the design of the study and performed the statistical analysis. LX participated in the preparation of JTW. JJ and SZ were involved in the determination of the main chemical constituents in JTW by 3D-HPLC analysis. XZ and WH were involved in the animal feeding and EEG recordings. KF, DW and KW carried out the biochemical indexes tests and PCR experiments. We thank Professor FL for helpful and professional advice. All authors read and approved the final manuscript.

\section{Competing interests}

The authors declare that they have no competing interests.

Consent for publication

Not applicable.

\section{Ethics approval}

All procedures were approved by Huazhong University of Science and Technology Ethics Committee for the use of experimental animals.

\section{Author details}

${ }^{1}$ Institute of Integrated Traditional Chinese and Western Medicine, Tongji Hospital, Tongji Medical College, Huazhong University of Science and Technology, Wuhan 430030, People's Republic of China. ${ }^{2}$ Shijiazhuang YiLing Pharmaceutical Co., Ltd., Shijiazhuang 050035, People's Republic of China. ${ }^{3}$ Department of Radiology, Tongji Hospital, Tongji Medical College, Huazhong University of Science and Technology, Wuhan 430030, People's Republic of China.

Received: 28 August 2016 Accepted: 23 February 2017

Published online: 23 March 2017

\section{References}

1. Shlisky JD, Hartman TJ, Kris-Etherton PM, Rogers CJ, Sharkey NA, NickolsRichardson SM. Partial sleep deprivation and energy balance in adults: an emerging issue for consideration by dietetics practitioners. J Acad Nutr Diet. 2012;112(11):1785-97.

2. Beccuti G, Pannain S. Sleep and obesity. Curr Opin Clin Nutr Metab Care. 2011;14(4):402-12

3. Leslie M. Circadian rhythms. Sleep study suggests triggers for diabetes and obesity. Science. 2012;336(6078):143.

4. Lucassen EA, Rother Kl, Cizza G. Interacting epidemics? Sleep curtailment, insulin resistance, and obesity. Ann N Y Acad Sci. 2012:1264:110-34.

5. Lou P, Chen P, Zhang L, Zhang P, Yu J, Zhang N, Wu H, Zhao J. Relation of sleep quality and sleep duration to type 2 diabetes: a population-based cross-sectional survey. BMJ Open. 2012;2(4):e000956.

6. Zimberg IZ, Damaso A, Del RM, Carneiro AM, de Sa SH, de Lira FS, Tufik S, de Mello MT. Short sleep duration and obesity: mechanisms and future perspectives. Cell Biochem Funct. 2012;30(6):524-9.

7. Carroll JE, Carrillo C, Olmstead R, Witarama T, Breen EC, Yokomizo M, Seeman T, Irwin MR. Sleep deprivation and divergent toll-like receptor-4 activation of cellular inflammation in aging. Sleep. 2015;38(2):205-11.

8. Chennaoui M, Sauvet F, Drogou C, Van Beers P, Langrume C, Guillard M, Gourby B, Bourrilhon C, Florence G, Gomez-Merino D. Effect of one night of sleep loss on changes in tumor necrosis factor alpha (TNF-alpha) levels in healthy men. Cytokine. 2011:56(2):318-24.

9. Herder C, Schneitler S, Rathmann W, Haastert B, Schneitler H, Winkler H, Bredahl R, Hahnloser E, Martin S. Low-grade inflammation, obesity, and insulin resistance in adolescents. J Clin Endocrinol Metab. 2007;92(12): 4569-74.

10. Hasnain M, Vieweg W. Weight considerations in psychotropic drug prescribing and switching. Postgrad Med. 2013;125(5):117-29.

11. Wang $H$, Quan S, Sheng Y. Effect of Jiaotai pill on serum cytokine in rats with insomnia induced by para-chloroamphetamine acid. Guangzhou Zhong Yi Yao Da Xue Xue Bao. 2008;25(6):525-7.

12. Chen $F$, Jong M, Chen $Y$, Kung $Y$, Chen T, Chen F, Hwang S. Prescriptions of Chinese herbal medicines for insomnia in Taiwan during 2002. Evid Based Compl Alt. 2011;2011:1-9. 
13. Yeung W, Chung K, Man-Ki Poon M, Yan-Yee Ho F, Zhang S, Zhang Z, Tat-Chi Ziea E, Wong VT. Chinese herbal medicine for insomnia: a systematic review of randomized controlled trials. Sleep Med Rev. 2012; 16(6):497-507.

14. Kou J, Wu Y, Wang Q, Yu B. Effect of raw Rhizome Coptidis or Rhizome Coptidis processed with wine on sedative and hypnotic effects of Jiao Tai Pills. Zhong Yao Yao Li Yu Lin Chuang. 2007;05:15-7.

15. Quan S, Zhang L, Lin X, Liu N. Study on the best compatibility of Jiao Tai Pills. He Nan Zhong Yi Xue Yuan Xue Bao. 2008;02:28-9.

16. Chen G, Lu F, Xu L, Dong H, Yi P, Wang F, Huang Z, Zou X. The anti-diabetic effects and pharmacokinetic profiles of berberine in mice treated with Jiao-Tai-Wan and its compatibility. Phytomedicine. 2013;20(10): $780-6$.

17. Huang Z, Xu X, Lu F, Wang N, Chen G, Zhao Y, Zou X, Wang K, Dong $\mathrm{H}, \mathrm{Xu}$ L. Jiao tai wan attenuates hepatic lipid accumulation in type 2 diabetes mellitus. Evid Based Complement Alternat Med. 2013;2013: 567045 .

18. Dong H, Wang JH, Lu FE, Xu LJ, Gong YL, Zou X. Jiaotai Pill enhances insulin signaling through phosphatidylinositol 3-kinase pathway in skeletal muscle of diabetic rats. Chin J Integr Med. 2013;19(9):668-74.

19. Hu N, Yuan L, Li HJ, Huang C, Mao QM, Zhang YY, Lin M, Sun YQ, Zhong XY, Tang $P$, et al. Anti-diabetic activities of Jiaotaiwan in $\mathrm{db} / \mathrm{db}$ mice by augmentation of AMPK protein activity and upregulation of GLUT4 expression. Evid Based Complement Alternat Med. 2013;2013: 180721.

20. He W, Liu G, Cai H, Sun X, Hou W, Zhang P, Xie Z, Liao Q. Integrated pharmacokinetics of five protoberberine-type alkaloids in normal and insomnic rats after single and multiple oral administration of Jiao-Tai-Wan. J Ethnopharmacol. 2014;154(3):635-44.

21. Levin BE. Arcuate NPY neurons and energy homeostasis in diet-induced obese and resistant rats. Am J Physiol. 1999;276(2 Pt 2):R382-7.

22. Mavanji V, Teske JA, Billington CJ, Kotz CM. Elevated sleep quality and orexin receptor mRNA in obesity-resistant rats. Int J Obes (Lond). 2010; 34(11):1576-88.

23. Mavanji V, Teske JA, Billington CJ, Kotz CM. Partial sleep deprivation by environmental noise increases food intake and body weight in obesityresistant rats. Obesity (Silver Spring). 2013;21(7):1396-405.

24. Mavanji V, Billington CJ, Kotz CM, Teske JA. Sleep and obesity: a focus on animal models. Neurosci Biobehav Rev. 2012;36(3):1015-29.

25. Cui XY, Cui SY, Zhang J, Wang ZJ, Yu B, Sheng ZF, Zhang XQ, Zhang YH Extract of Ganoderma lucidum prolongs sleep time in rats. J Ethnopharmacol. 2012;139(3):796-800

26. Gao J, Zhang JX, Xu TL.Modulation of serotonergic projection from dorsal raphe nucleus to basolateral amygdala on sleep-waking cycle of rats. Brain research 2002;945(1):60-70. doi10.1016/50006-8993(02)02625-2

27. Lavebratt $C$, Sjoholm LK, Soronen P, Paunio T, Vawter MP, Bunney WE, Adolfsson R, Forsell $Y$, Wu JC, Kelsoe JR, et al. CRY2 is associated with depression. PLoS One. 2010;5(2):e9407.

28. Narasimamurthy R, Hatori M, Nayak SK, Liu F, Panda S, Verma IM. Circadian clock protein cryptochrome regulates the expression of proinflammatory cytokines. Proc Natl Acad Sci U S A. 2012;109(31):12662-7.

29. Tak PP, Firestein GS. NF-kappaB: a key role in inflammatory diseases. J Clin Invest. 2001;107(1):7-11.

30. Tanti JF, Ceppo F, Jager J, Berthou F. Implication of inflammatory signaling pathways in obesity-induced insulin resistance. Front Endocrinol (Lausanne). 2012;3:181.

31. Dibner $\mathrm{C}$, Schibler U, Albrecht U. The mammalian circadian timing system: organization and coordination of central and peripheral clocks. Annu Rev Physiol. 2010;72:517-49.

32. Liu AC, Lewis WG, Kay SA. Mammalian circadian signaling networks and therapeutic targets. Nat Chem Biol. 2007;3(10):630-9.

33. Tasali E, Leproult R, Ehrmann DA, Van Cauter E. Slow-wave sleep and the risk of type 2 diabetes in humans. Proc Natl Acad Sci U S A. 2008;105(3): 1044-9.

34. Boivin DB, James FO, Wu A, Cho-Park PF, Xiong H, Sun ZS. Circadian clock genes oscillate in human peripheral blood mononuclear cells. Blood. 2003; 102(12):4143-5.
35. Emanuela F, Grazia M, Marco DR, Maria PL, Giorgio F, Marco B. Inflammation as a link between obesity and metabolic syndrome. J Nutr Metab. 2012; 2012:476380.

36. Lee $\mathrm{YH}$, Pratley RE. The evolving role of inflammation in obesity and the metabolic syndrome. Curr Diab Rep. 2005;5(1):70-5.

\section{Submit your next manuscript to BioMed Central and we will help you at every step:}

- We accept pre-submission inquiries

- Our selector tool helps you to find the most relevant journal

- We provide round the clock customer support

- Convenient online submission

- Thorough peer review

- Inclusion in PubMed and all major indexing services

- Maximum visibility for your research

Submit your manuscript at www.biomedcentral.com/submit
Biomed Central 